\title{
Age-Related Physiologic Thinning Rate of the Retinal Nerve Fiber Layer in Different Levels of Myopia
}

\author{
Daun Jeong, ${ }^{1}$ Kyung Rim Sung $\mathbb{D}^{1},{ }^{1}$ Youn Hye Jo, ${ }^{1}$ and Sung-cheol Yun ${ }^{2}$ \\ ${ }^{1}$ Department of Ophthalmology, College of Medicine, University of Ulsan, Asan Medical Center, Seoul, Republic of Korea \\ ${ }^{2}$ Department of Clinical Epidemiology and Biostatistics, Department of Ophthalmology, College of Medicine, University of Ulsan, \\ Asan Medical Center, Seoul, Republic of Korea \\ Correspondence should be addressed to Kyung Rim Sung; sungeye@gmail.com
}

Received 20 July 2019; Revised 29 October 2019; Accepted 7 November 2019; Published 21 January 2020

Academic Editor: Sentaro Kusuhara

Copyright (C) 2020 Daun Jeong et al. This is an open access article distributed under the Creative Commons Attribution License, which permits unrestricted use, distribution, and reproduction in any medium, provided the original work is properly cited.

\begin{abstract}
Purpose. To investigate the effect of refractive error on the physiologic thinning rate of the retinal nerve fiber layer (RNFL) in healthy eyes. Materials and Methods. This study analyzed 223 eyes of 141 healthy subjects followed for more than 5 years and underwent at least five serial spectral domain optical coherence tomography (SD-OCT) examinations. Longitudinal RNFL measurements were analyzed by linear mixed models incorporating follow-up duration, baseline RNFL thickness, spherical equivalent (SE), age, intraocular pressure, and visual field mean deviation. Thinning rates were classified according to SE into three groups: nonmyopic (NM; >0 D), mild-to-moderately myopic (MM; >-6 D and $\leq 0 \mathrm{D})$, and highly myopic (HM; $\leq-6 \mathrm{D})$. Results. The overall slopes of change in RNFL thickness over time in the NM, MM, and HM groups were $-0.305 \pm 0.128,-0.294 \pm 0.068$, and $-0.208 \pm 0.097 \mu \mathrm{m} / \mathrm{yr}$, respectively. Slopes of RNFL thickness changes in these groups were $-0.514 \pm 0.248,-0.520 \pm 0.133$, and $-0.528 \pm 0.188 \mu \mathrm{m} / \mathrm{yr}$, in the superior quadrant; $-0.084 \pm 0.145,0.107 \pm 0.082$, and $-0.161 \pm 0.112 \mu \mathrm{m} / \mathrm{yr}$, in the temporal quadrant; $-0.807 \pm 0.242,-0.794 \pm 0.130$, and $-0.727 \pm 0.183 \mu \mathrm{m} / \mathrm{yr}$, in the inferior quadrant; and $0.160 \pm 0.157,0.118 \pm 0.084$, and $0.429 \pm 0.119 \mu \mathrm{m} / \mathrm{yr}$, in the nasal quadrant. Overall and in all four quadrants, there was no significant difference in the rate of RNFL thickness change among the three groups. Conclusions. Refractive error did not affect the physiologic thinning rate of RNFL when assessed by SD OCT.
\end{abstract}

\section{Introduction}

Glaucoma is a progressive optic neuropathy, which can lead to blindness in the absence of timely treatment. Although optimal treatment requires a determination of glaucomatous deterioration, this determination is hindered by the concomitant occurrence of age-associated physiologic deterioration. Normal subjects have shown age-associated progressive thinning of their retinal nerve fiber layer (RNFL) and reductions in visual field (VF) sensitivity [1-7]. Thus, in defining glauomatous progression, it is important to consider this physiologic worsening rate, such that progressive changes exceeding the physiologic thinning rate can be regarded as pathologic glaucomatous deterioration.
Myopia is a risk factor for primary open angle glaucoma (POAG), and a high proportion of patients with POAG in clinic is myopic [8-11]. Hence, understanding the characteristics of myopic eyes is essential for the treatment of myopic POAG patients. Although RNFL is thinner in myopic than in nonmyopic eyes $[12,13]$, it remains unclear, whether the physiologic thinning rate of the RNFL is faster in myopic than in nonmyopic eyes. If myopic eyes have a faster physiologic thinning rate, this rate should be considered when determining glaucomatous progression in myopic POAG patients. Therefore, the current study assessed the longitudinal RFNL thinning rates in normal healthy eyes as a function of refractive error, as determined by spectral domain optical coherence tomography (SD-OCT). 


\section{Methods}

2.1. Subjects. Subjects at the Asan Medical Center who underwent ocular health screening and were followed up by Cirrus HD-OCT (Carl Zeiss Meditec Inc., Dublin, CA, USA) at least five times for more than 5 years between January 2009 and March 2018 were included in this study. The medical records of these subjects were reviewed retrospectively by a single glaucoma specialist (DJ). Initial testing included a comprehensive ophthalmological examination, including a review of medical history, measurement of bestcorrected visual acuity (BCVA), slit lamp biomicroscopy, and multiple measurements of intraocular pressure (IOP) by Goldmann applanation tonometry, gonioscopy, dilated funduscopic examination, stereoscopic optic disc photography, red-free RNFL photography, standard automated perimetry (SITA Standard 24-2; Carl Zeiss Meditec Inc.), and Cirrus HD-OCT.

All eyes had a BCVA of $\geq 20 / 30$ with a normal anterior chamber and normal retinae on slit lamp examination and fundus photography. Subjects with an abnormal appearing optic disc or an RNFL defect were excluded after careful review of the optic disc/RNFL photography results by two glaucoma specialists (KRS and DJ). Also excluded were subjects who were eligible at initial testing but who later developed a VF or RNFL defect or a glaucomatous optic disc during the follow-up period. Eyes with retinal pathology, such as an epiretinal membrane or diabetic retinopathy, were also excluded. Eyes with a history of refractive surgery, intraocular surgery, or retinal laser therapy at initial work up, as well as those that underwent these procedures any time during the follow-up period were also excluded. Hence, eyes that were aphakic or pseudophakic at baseline examination were also excluded.

Eyes were divided into the three groups based on the level of baseline spherical equivalent (SE): nonmyopic (NM, $>0 \mathrm{D})$, mild-to-moderate myopic (MM, $>-6 \mathrm{D}<$ and $\leq 0 \mathrm{D})$, and highly myopic (HM; $\leq-6 \mathrm{D})$. The study protocol was approved by the Institutional Review Board of the Asan Medical Center, and the study was performed in accordance with principles of the Declaration of Helsinki.

2.2. SD-OCT Imaging. SD-OCT images were obtained as described using a Cirrus HD-OCT system, which is calibrated regularly by a technician employed by the manufacturer [14-16]. RNFL thicknesses were obtained using the optic disc cube mode. All included eyes exhibited a centered optic disc and were well focused with even and adequate illumination, did not move within the measurement circle and had a signal strength of at least seven. RNFL thickness was measured in four quadrants (temporal, superior, nasal, and inferior). For inclusion in analysis, at least five acceptable SD-OCT images of each eye, taken at separate visits, were required.

2.3. Statistical Analysis. Continuous variables are reported as mean \pm standard deviation and categorical variables as number (frequency). The overall progression rates of RNFL thickness were determined from serial measurements of each parameter using a linear mixed effect model (LMM). Models were fitted with fixed coefficients (fixed effects) of participant baseline RNFL thickness, SE, age, gender, IOP measurements, visual field mean deviation (VF MD), time (i.e., follow-up duration), and the interactions of SE, age, gender, IOP, VF MD, and follow-up duration with random intercepts. Residual diagnostic plots were used to detect features of concern in the model. Exploratory analyses of the residuals suggested that the chosen models were appropriate. The adjusted slopes of the average RNFL thicknesses and of the RNFL thicknesses in each quadrant were compared among the NM, MM, and HM groups using a testing interaction term in the LMM. All reported $P$ values were two-sided, and a value of $P<0.05$ was considered statistically significant. All statistical analyses were performed using SAS software, version 9.2 (SAS Institute, Inc, Cary, NC).

\section{Results}

Over a mean follow-up period of approximately 6 years, 10 eyes of nine subjects who were eligible for inclusion at baseline examination developed glaucomatous optic disc changes and were therefore excluded. Thus, 1407 images of 223 eyes of 141 healthy subjects were analyzed. All 141 subjects were East Asians (Koreans), including 74 (52.5\%) men and 67 (47.5\%) women. Of the 141 subjects, $11(7.8 \%)$ had diabetes mellitus, $30(21 \%)$, systemic hypertension, and $9(6 \%)$ had a family history of glaucoma. Of the 223 eyes, 37 (16.6\%), $124(55.6 \%)$, and 62 (27.8\%) were classified by SE into the NM, MM, and HM groups, making $83.4 \%$ of all eyes myopic. Characteristics of the study participants are summarized in Table 1. Each eye underwent an average of 6.31 OCT examinations, and the mean duration of follow-up was $5.8 \pm 0.7$ years. At baseline, the mean SE was $-4.0 \pm 3.6 \mathrm{D}$ and the mean IOP was $16 \pm 2.9 \mathrm{mmHg}$, with a mean follow-up IOP of $15 \pm 2.3 \mathrm{mmHg}$. Mean VF MD at baseline was $-0.79 \pm 1.24 \mathrm{~dB}$, and mean RNFL thickness was $85.8 \pm 9.8 \mu \mathrm{m}$. Table 2 shows the baseline demographic and clinical characteristics of the three subgroups based on SE. Age at baseline was higher in the NM than in the MM and HM groups.

The change of RNFL thickness over time in all 223 eyes was estimated using an LMM (Table 3), which showed a significant interaction between thinning rate and time. Overall slope over time was $-0.389 \mu \mathrm{m} / \mathrm{yr}(P<0.0001)$ in men and $-0.164 \mu \mathrm{m} / \mathrm{yr}$ in women, a difference of $0.225 \mu \mathrm{m} / \mathrm{yr}$ that was statistically significant $(P=0.006)$. The slopes of RNFL thickness in the superior, temporal, and inferior quadrants, but not in the nasal quadrant, showed significant interactions with time. The changes in slope over time were $-0.524 \mu \mathrm{m} / \mathrm{yr}$ in the superior sector and $-0.778 \mu \mathrm{m} / \mathrm{yr}$ in the inferior sector, with none of the analyzed variables influencing these slopes. In the temporal sector, the RNFL thickness was $0.034 \mu \mathrm{m} / \mathrm{yr}$ less reduced as myopia increased by one diopter, resulting in a rate of $-0.316(-0.034)=-0.282 \mu \mathrm{m} / \mathrm{yr}(P=0.040)$, with the rate of reduction being $0.345 \mu \mathrm{m} /$ year greater in men than in women $(P=0.006)$. 
TABle 1: Demographics and optical coherence tomography circumpapillary retinal nerve fiber layer (RNFL) and macular measurements.

\begin{tabular}{lc}
\hline Subjects & 223 eyes from 141 individuals \\
\hline Age (yrs) & $40 \pm 12$ \\
Gender (male: female) & $111: 112$ \\
Mean f/u frequency & $6.31 \pm 1.22$ \\
Mean f/u period (yrs) & $5.8 \pm 0.71$ \\
S.E (D) & $-4.0 \pm 3.6$ \\
Baseline IOP (mmHg) & $16 \pm 2.9$ \\
F/u mean IOP (mmHg) & $15 \pm 2.3$ \\
Baseline MD (dB) & $-0.79 \pm 1.24$ \\
avr. RNFL thickness $(\mu \mathrm{m})$ & $85.8 \pm 9.81$ \\
& Of 141 individuals \\
DM (\%) & $7.8(n=11)$ \\
HTN (\%) & $21(n=30)$ \\
Family history of glaucoma $(\%)$ & $6(n=9)$ \\
\hline
\end{tabular}

S.E: sepherical equivalent; IOP: intraocular pressure; f/u: follow-up; RNFL: retinal nerve fiber layer; GCIPL: ganaglion cell-inner plexiform layer.

We compared the rate of changes in RNFL thickness in the three SE groups after adjusting for between-eye interactions within each subject, sex, age, SE, IOP, mean followup IOP, baseline RNFL thickness, VF MD, and laterality (Table 4). The overall slopes of change in RNFL thickness over time in the NM, MM, and HM groups were $-0.305 \pm 0.128,-0.294 \pm 0.068$, and $-0.208 \pm 0.097 \mu \mathrm{m} / \mathrm{yr}$, respectively. The slopes of RNFL thickness changes in these groups were $-0.514 \pm 0.248,-0.520 \pm 0.133$, and $-0.528 \pm 0.188 \mu \mathrm{m} / \mathrm{yr}$, respectively, in the superior quadrant; $-0.084 \pm 0.145,0.107 \pm 0.082$, and $-0.161 \pm 0.112 \mu \mathrm{m} / \mathrm{yr}$, respectively, in the temporal quadrant; $-0.807 \pm 0.242$, $-0.794 \pm 0.130$, and $-0.727 \pm 0.183 \mu \mathrm{m} / \mathrm{yr}$, respectively, in the inferior quadrant; and $0.160 \pm 0.157,0.118 \pm 0.084$, and $0.429 \pm 0.119 \mu \mathrm{m} / \mathrm{yr}$, respectively in the nasal quadrant. Overall and in each quadrant, there was no significant difference in the rate of RNFL thickness change among the three SE-based groups.

\section{Discussion}

This longitudinal analysis of 223 healthy eyes for approximately 6 years showed that average RNFL thickness decreased at rates of $0.208-0.305 \mu \mathrm{m} / \mathrm{yr}$ after adjustment for covariates. In comparison, cross-sectional studies that included subjects of various ages found that average RNFL thickness decreased at rates of $0.16-0.26 \mu \mathrm{m} / \mathrm{yr}$, slightly less than in our study $[1,3,4,17]$. However, a longitudinal study that investigated RNFL changes in normal eyes of Hong Kong Chinese, found that the rate of average RNFL thickness change was $-0.33 \mu \mathrm{m} / \mathrm{yr}$ [2], and a study similar to ours reported a rate of $-0.54 \mu \mathrm{m} / \mathrm{yr}$ [6], Thus, in general, thinning rates have been higher in longitudinal than in cross-sectional studies. Cross-sectional studies, however, cannot determine the true rate of change over time in the same participants; rather, they compare slopes of RNFL thickness in various age groups. Thus, the actual age-associated rate of reduction of RNFL thickness in normal eyes is approximately $0.3 \mu \mathrm{m} / \mathrm{yr}$.
RNFLs are thinner in myopic than in nonmyopic eyes, with thickness associated with the degree of myopia $[13,18,19]$. Although baseline RNFL in our study subjects was thinnest in the HM group, refractive error was not associated with the overall thinning rate of RNFL during follow-up. Moreover, a comparison of the adjusted slopes of RNFL change showed no significant differences among the three SE-based groups. The reason for the inverse association between RNFL thickness and degree of myopia is unclear. However, myopic axial elongation may subject retinal nerve fibers to tensile stress, which may eventually damage the retinal nerve fibers and reduce RNFL thickness. Because this myopic axial elongation usually occurs during childhood or early adolescence, the normal thinning rate may not have been affected by myopic axial elongation in our participants, whose mean baseline age was 40 years. In addition, myopic glaucomatous eyes have thinner RNFLs, with myopia being a risk factor for glaucoma development [20, 21]. However, myopia was not associated with faster progression of glaucoma in these studies, although previous studies have yielded inconsistent results [22]. Myopic axial elongation may damage the RNFL, thereby affecting the development of glaucoma in some young patients $[8,20,23,24]$. However, because myopic elongation stops at adolescence, subjects with myopic glaucoma may not show rapid deterioration [25]. Similarly, our results may suggest that although the RFNL tended to be thinner in healthy myopic than in healthy nonmyopic eyes and that RNFL tended to be thinner as the degree of myopia increases, these myopic eyes do not show a more rapid age-related rate of RNFL thinning because myopic elongation stops or slows when one becomes an adult.

Examination of the four quadrants of the eyes included in this study showed that thinning rate was fastest in the inferior quadrant $(-0.778 \mu \mathrm{m} / \mathrm{yr})$, followed by the superior quadrant $(-0.524 \mu \mathrm{m} / \mathrm{yr})$. These quadrants were previously reported to have faster thinning rates than the nasal and temporal quadrants [2]. The faster rate of RNFL thinning in the superior and inferior quadrants may be due to the structural weakness of these areas in the optic nerve head lamina cribrosa. The superior and inferior regions of the lamina cribrosa have been reported weaker, making these regions more vulnerable to glaucomatous damage $[22,26]$. This relative weakness of support tissue can also affect physiologic thinning in healthy eyes.

In contrast to findings in the inferior and superior quadrants, the nasal quadrant showed no significant changes over time in RNFL thickness. Similarly, thickening of the RNFL in this quadrant by $+0.308 \mu \mathrm{m} / \mathrm{yr}$ has been reported [2], further suggesting that the nasal sector did not experience age-related loss in RNFL thickness. This may have been due to the proportion of nonneuronal tissue, such as glial tissue, in the RNFL, which has been reported to increase with age $[27,28]$. OCT assesses the thickness between the internal limiting membrane and the ganglion cell layer in the retina; thus, OCT cannot measure the RNFL separately from other layers. Axonal fibers in the RNFL decrease with age, indicating an inverse relationship between thickness and the proportion of nonneuronal tissue. Thus, changes in RNFL, 
TABLE 2: Clinical characteristics of three subgroups based on SE.

\begin{tabular}{|c|c|c|c|c|c|c|}
\hline \multirow[b]{2}{*}{ Age (yrs) } & \multirow{2}{*}{$\begin{array}{c}\text { NMG (A), } n=37 \\
50 \pm 13\end{array}$} & \multirow{2}{*}{$\begin{array}{c}\text { MMG (B), } n=124 \\
39 \pm 11\end{array}$} & \multirow{2}{*}{$\begin{array}{c}\text { HMG (B), } n=62 \\
36 \pm 12\end{array}$} & \multicolumn{3}{|c|}{$\begin{array}{c}P \text { value (A vs. } \mathrm{B}, \mathrm{A} \text { vs. } \mathrm{C}, \\
\text { B vs. } \mathrm{C} \text { ) }\end{array}$} \\
\hline & & & & $<0.001$ & $<0.001$ & 0.133 \\
\hline Gender (male:female) & $12: 25$ & $70: 54$ & $29: 33$ & $0.354^{I}$ & & \\
\hline S.E (D) & $0.69 \pm 0.79$ & $-2.96 \pm 1.69$ & $-8.7 \pm 1.77$ & $<0.001$ & $<0.001$ & $<0.001^{*}$ \\
\hline Baseline IOP (mmHg) & $15 \pm 3.7$ & $16 \pm 3.0$ & $16 \pm 2.2$ & 1.0 & 1.0 & 1.0 \\
\hline Follow-up mean IOP (mmHg) & $14 \pm 2.2$ & $15 \pm 2.5$ & $15 \pm 2.1$ & 0.373 & 0.207 & 1 \\
\hline Baseline MD (dB) & $-0.47 \pm 1.3$ & $-0.74 \pm 1.2$ & $-1.1 \pm 1.3$ & 0.733 & 0.059 & 0.253 \\
\hline Global RNFL thickness $(\mu \mathrm{m})$ & $90.2 \pm 9.5$ & $86.9 \pm 10.3$ & $82.1 \pm 7.6$ & 0.108 & $<0.001$ & 0.009 \\
\hline RNFL thickness, superior quad. $(\mu \mathrm{m})$ & $113.1 \pm 17.1$ & $107.4 \pm 17.4$ & $100.0 \pm 13.8$ & 0.207 & 0.001 & 0.017 \\
\hline RNFL thickness, temporal quad. $(\mu \mathrm{m})$ & $67.4 \pm 8.3$ & $67.5 \pm 12.5$ & $71.8 \pm 14.0$ & 1.0 & 0.281 & 0.089 \\
\hline RNFL thickness, inferior quad. $(\mu \mathrm{m})$ & $118.2 \pm 16.7$ & $110.0 \pm 17.6$ & $99.0 \pm 13.5$ & 0.02 & $<0.001$ & $<0.001$ \\
\hline RNFL thickness, nasal quad. $(\mu \mathrm{m})$ & $62.2 \pm 8.4$ & $62.9 \pm 9.7$ & $57.5 \pm 9.0$ & 1.0 & 0.056 & 0.001 \\
\hline
\end{tabular}

SE: spherical equivalent, $\mathrm{D}=$ diopters; $\mathrm{IOP}=$ intraocular pressure; $\mathrm{MD}=$ mean deviation; $\mathrm{RNFL}=$ retinal nerve fiber layer, quad. $=$ quadrant. $\mathrm{Data}$ are presented as mean values \pm standard deviation. Comparison among three groups was performed by analysis of variance with the Bonferroni method. ${ }^{I} P$ value with the linear-by-linear association test. ${ }^{*} P$ values with the Dunnett T3 method.

TABLE 3: Retinal nerve fiber layer thinning rates $(\mu \mathrm{m} /$ year $)$ calculated by linear mixed effect model.

\begin{tabular}{|c|c|c|c|c|c|c|c|c|c|c|c|c|c|c|c|}
\hline & \multicolumn{3}{|c|}{ Average of all sectors } & \multicolumn{3}{|c|}{ Superior sector } & \multicolumn{3}{|c|}{ Temporal sector } & \multicolumn{3}{|c|}{ Inferior sector } & \multicolumn{3}{|c|}{ Nasal sector } \\
\hline & Beta & SE & $\begin{array}{c}P \\
\text { value }\end{array}$ & Beta & SE & $\begin{array}{c}P \\
\text { value }\end{array}$ & Beta & SE & $\begin{array}{c}P \\
\text { value }\end{array}$ & Beta & SE & $\begin{array}{c}P \\
\text { value }\end{array}$ & Beta & SE & $\begin{array}{c}P \\
\text { value }\end{array}$ \\
\hline Intercept & 102.170 & 5.404 & $<.0001$ & 133.850 & 9.958 & $<.0001$ & 88.397 & 6.739 & $<.0001$ & 140.350 & 9.829 & $<.0001$ & 42.930 & 5.590 & $<.0001$ \\
\hline age (years) & -0.206 & 0.069 & 0.003 & -0.417 & 0.111 & $<.0001$ & -0.250 & 0.088 & 0.005 & -0.327 & 0.123 & 0.008 & 0.139 & 0.068 & 0.041 \\
\hline Female & 4.279 & 1.528 & 0.005 & 4.213 & 2.394 & 0.079 & 5.799 & 2.024 & 0.004 & 9.122 & 2.683 & 0.001 & -0.834 & 1.470 & 0.571 \\
\hline Right eye & 0.371 & 0.252 & 0.142 & -3.260 & 0.485 & $<.0001$ & 3.007 & 0.311 & $<.0001$ & -0.206 & 0.483 & 0.670 & 1.988 & 0.293 & $<.0001$ \\
\hline $\begin{array}{l}\text { Spherical } \\
\text { equivalent (D) }\end{array}$ & 0.287 & 0.176 & 0.103 & 1.471 & 0.299 & $<.0001$ & 37 & 0.220 & $<.0001$ & 0.884 & 0.320 & 0.006 & 0.199 & .182 & 0.275 \\
\hline $\begin{array}{l}\text { Baseline IOP } \\
(\mathrm{mmHg})\end{array}$ & -0.081 & 0.223 & 0.716 & -0.184 & 0.410 & 0.654 & -0.953 & 0.275 & 0.001 & 0.212 & 0.420 & 0.614 & 0.573 & 0.249 & 0.021 \\
\hline $\begin{array}{l}\text { Mean F/U IOP } \\
(\mathrm{mmHg})\end{array}$ & -0.512 & 0.350 & 0.144 & -0.443 & 0.622 & 0.477 & -0.333 & 0.433 & 0.443 & -1.540 & 0.650 & 0.018 & 0.322 & 0.379 & 0.396 \\
\hline VF MD (dB) & 0.504 & 0.196 & 0.010 & 1.054 & 0.370 & 0.005 & -0.336 & 0.241 & 0.164 & 1.426 & 0.373 & $<.0001$ & 0.286 & 0.267 & 0.285 \\
\hline Time & -0.389 & 0.074 & $<.0001$ & -0.524 & 0.100 & $<.0001$ & -0.316 & 0.110 & 0.005 & -0.778 & 0.099 & $<.0001$ & 0.121 & 0.073 & 0.100 \\
\hline Female & 0.225 & 0.103 & 0.030 & - & - & - & 0.345 & 0.124 & 0.006 & - & - & - & - & - & - \\
\hline Age (years) & - & - & - & - & - & - & - & - & - & - & - & - & - & - & - \\
\hline $\begin{array}{l}\text { Spherical } \\
\text { equivalent (D) }\end{array}$ & - & - & - & - & - & - & -0.034 & 0.017 & 0.040 & - & - & - & - & - & - \\
\hline $\begin{array}{l}\text { Mean F/U IOP } \\
(\mathrm{mmHg})\end{array}$ & - & - & - & - & - & - & - & - & - & - & - & - & - & - & - \\
\hline $\operatorname{VF} M D(d B)$ & - & - & - & - & - & - & - & - & - & - & - & - & -0.116 & 0.048 & 0.017 \\
\hline
\end{tabular}

SE: standard error; D: diopter; IOP: intraocular pressure; MD: mean deviation.

TABle 4: Rates of change in RNFL thickness of the three myopia groups based on spherical equivalent.

\begin{tabular}{lccccccc}
\hline & \multicolumn{2}{c}{ NM } & \multicolumn{2}{c}{ MM } & \multicolumn{2}{c}{ HM } & \multirow{2}{*}{$P$ value } \\
& Slope & SE & Slope & SE & Slope & SE & \\
\hline Superior & -0.514 & 0.248 & -0.520 & 0.133 & -0.528 & 0.188 & 0.999 \\
Temporal & -0.084 & 0.145 & 0.107 & 0.082 & -0.161 & 0.112 & 0.111 \\
Inferior & -0.807 & 0.242 & -0.794 & 0.130 & -0.727 & 0.183 & 0.947 \\
Nasal & 0.160 & 0.157 & 0.118 & 0.084 & 0.429 & 0.119 & 0.094 \\
Average & -0.305 & 0.128 & -0.294 & 0.068 & -0.208 & 0.097 & 0.738 \\
\hline
\end{tabular}

NM: nonmyopic; MM: mild-to-moderately myopic; HMG: highly myopic.

as measured by OCT, result from a combination of a decreased width of neuronal tissue and an increased width of nonneuronal tissue. This would apply not only to the nasal quadrant but also to all quadrants. However, consistent results showing that RNFL thickness in the nasal sector increases or remains stable suggest an effect of nonneuronal tissue and its possible increase over time.

In contrast to our results, RNFL thinning rate has been reported to be faster in highly myopic than in nonmyopic eyes in subjects aged 40-59 years who were followed-up for more than 3 years [29]. Because our subjects were relatively younger (mean age, $40 \pm 12$ years), direct comparison between these studies may be inappropriate. Furthermore, the previous study also reported that RNFL thinning rates were similar in highly myopic and nonmyopic eyes of younger subjects, in agreement with our results [29]. Furthermore, the shorter follow-up period in the previous study (3 years) than in ours (6 years) may have resulted in different outcomes. Highly myopic eyes with injured and thinner RNFL in younger subjects due to a sudden increase in axial length may experience a faster deterioration of the RNFL as the subject becomes 40-59 years old. Further studies are needed, 
however, to test this hypothesis. Our participants showed faster thinning in men compared with women. This may be due to thinner baseline RNFL in men than in women (83.4 vs. $87.7 \mu \mathrm{m})$.

To our knowledge, this is the first study to compare RNFL changes as a function of refractive error in healthy eyes for a relatively long period of time. Although myopic eyes are more vulnerable to glaucomatous changes [30-33], we found that, after controlling for confounding variables, refractive error had no impact on RNFL thickness change in normal eyes.

This study had several limitations. Our population consisted of normal subjects; nonetheless, it was a retrospective analysis of participants who had been followed-up annually during routine check-ups. All eyes with abnormally appearing optic discs were excluded. However, our participants may not represent the normal population. Moreover, all of the subjects in our study were East Asian, an ethnic population with a high prevalence of myopia, indicating the need to assess the association between myopia and the rate of change in RNFL thickness in other ethnic groups [34, 35]. In addition, we originally intended to investigate the relationship between axial length and age-related RNFL loss. However, as axial length was not measured in some participants, we assessed SE rather than axial length.

In summary, this study assessed the rate of age-related RNFL loss in normal, nonglaucomatous eyes. Refractive error did not affect the physiologic thinning of the RNFL, as assessed by SD OCT.

\section{Data Availability}

The data are available on reasonable request from the corresponding author.

\section{Conflicts of Interest}

The authors declare that they have no conflicts of interest.

\section{Acknowledgments}

This study was supported by a grant (2018-0303) from the Asan Institute for Life Sciences, Asan Medical Center, Seoul, South Korea, and by the Basic Science Research Program through the National Research Foundation of Korea (NRF), which was funded by the Ministry of Education, Science, and Technology (no. NRF-2017R1A2B4007792).

\section{References}

[1] R. S. Parikh, S. R. Parikh, G. C. Sekhar, S. Prabakaran, J. G. Babu, and R. Thomas, "Normal age-related decay of retinal nerve fiber layer thickness," Ophthalmology, vol. 114, no. 5, pp. 921-926, 2007.

[2] C. K. S. Leung, M. Yu, R. N. Weinreb et al., "Retinal nerve fiber layer imaging with spectral-domain optical coherence tomography," Ophthalmology, vol. 119, no. 4, pp. 731-737, 2012.

[3] D. L. Budenz, D. R. Anderson, R. Varma et al., "Determinants of normal retinal nerve fiber layer thickness measured by Stratus OCT," Ophthalmology, vol. 114, no. 6, pp. 1046-1052, 2007.
[4] H. Hirasawa, A. Tomidokoro, M. Araie et al., "Peripapillary retinal nerve fiber layer thickness determined by spectraldomain optical coherence tomography in ophthalmologically normal eyes," Archives of Ophthalmology, vol. 128, no. 11, pp. 1420-1426, 2010.

[5] T. Mansoori and N. Balakrishna, "Effect of aging on retinal nerve fiber layer thickness in normal asian indian eyes: a longitudinal study," Ophthalmic Epidemiology, vol. 24, no. 1, pp. 24-28, 2017.

[6] Z. Wu, L. J. Saunders, L. M. Zangwill, F. B. Daga, J. G. Crowston, and F. A. Medeiros, "Impact of normal aging and progression definitions on the specificity of detecting retinal nerve fiber layer thinning," American Journal of Ophthalmology, vol. 181, pp. 106-113, 2017.

[7] P. G. D. Spry and A. C. A. Johnson, "Senescent changes of the normal visual field: an age-old problem," Optometry and Vision Science, vol. 78, no. 6, pp. 436-441, 2001.

[8] M. W. Marcus, M. M. de Vries, F. G. J. Montolio, and N. M. Jansonius, "Myopia as a risk factor for open-angle glaucoma: a systematic review and meta-analysis," Ophthalmology, vol. 118, no. 10, pp. 1989-1994, 2011.

[9] S. Kim and K. R. Sung, "Factors associated with loss of visual function in medically treated advanced normal tension glaucoma," Current Eye Research, vol. 42, no. 3, pp. 429-435, 2017.

[10] J. Y. Lee, K. R. Sung, S. Han, and J. H. Na, "Effect of myopia on the progression of primary open-angle glaucoma," Investigative Ophthalmology \& Visual Science, vol. 56, no. 3, pp. 1775-1781, 2015.

[11] B. Chon, M. Qiu, and S. C. Lin, "Myopia and glaucoma in the South Korean population," Investigative Opthalmology \& Visual Science, vol. 54, no. 10, pp. 6570-6577, 2013.

[12] Z. Zhao and C. Jiang, "Effect of myopia on ganglion cell complex and peripapillary retinal nerve fibre layer measurements: a Fourier-domain optical coherence tomography study of young Chinese persons," Clin Exp Ophthalmol, vol. 41, no. 41, pp. 561-566, 2013.

[13] C. K.-S. Leung, S. Mohamed, K. S. Leung et al., "Retinal nerve fiber layer measurements in myopia: an optical coherence tomography study," Investigative Opthalmology \& Visual Science, vol. 47, no. 12, pp. 5171-5176, 2006.

[14] K. R. Sung, J. H. Sun, J. H. Na, J. Y. Lee, and Y. Lee, "Progression detection capability of macular thickness in advanced glaucomatous eyes," Ophthalmology, vol. 119, no. 2, pp. 308-313, 2012

[15] S. B. Park, K. R. Sung, S. Y. Kang et al., "Comparison of glaucoma diagnostic Capabilities of Cirrus HD and Stratus optical coherence tomography," Archives of Ophthalmology, vol. 127, no. 12, pp. 1603-1609, 2009.

[16] K. R. Sung, D. Y. Kim, S. B. Park, and M. S. Kook, "Comparison of retinal nerve fiber layer thickness measured by Cirrus HD and Stratus optical coherence tomography," Ophthalmology, vol. 116, no. 7, pp. 1264-1270, 2009.

[17] K. R. Sung, G. Wollstein, R. A. Bilonick et al., "Effects of age on optical coherence tomography measurements of healthy retinal nerve fiber layer, macula, and optic nerve head," Ophthalmology, vol. 116, no. 6, pp. 1119-1124, 2009.

[18] F. M. Rauscher, N. Sekhon, W. J. Feuer, and D. L. Budenz, "Myopia affects retinal nerve fiber layer measurements as determined by optical coherence tomography," Journal of Glaucoma, vol. 18, no. 7, pp. 501-505, 2009.

[19] M. J. Kim, E. J. Lee, and T.-W. Kim, "Peripapillary retinal nerve fibre layer thickness profile in subjects with myopia measured using the Stratus optical coherence tomography," 
British Journal of Ophthalmology, vol. 94, no. 1, pp. 115-120, 2010.

[20] T. W. Kim, M. Kim, R. N. Weinreb et al., "Optic disc change with incipient myopia of childhood," Ophthalmology, vol. 119, no. 1, pp. 21-26 e3, 2012.

[21] J. E. Lee, K. R. Sung, J. M. Park et al., "Optic disc and peripapillary retinal nerve fiber layer characteristics associated with glaucomatous optic disc in young myopia," Graefe's Archive for Clinical and Experimental Ophthalmology, vol. 255, no. 3, pp. 591-598, 2017.

[22] J. Y. Lee, K. R. Sung, and S.-C. Yun, "Comparison of rates of retinal nerve fibre layer thinning between patients with nonmyopic and myopic glaucoma," British Journal of Ophthalmology, vol. 100, no. 5, pp. 699-703, 2016.

[23] S. Lee, S. X. Han, M. Young, M. F. Beg, M. V. Sarunic, and P. J. Mackenzie, "Optic nerve head and peripapillary morphometrics in myopic glaucoma," Investigative Opthalmology \& Visual Science, vol. 55, no. 7, pp. 4378-4394, 2014.

[24] H. W. Bae, S. J. Seo, S. Y. Lee et al., "Risk factors for visual field progression of normal-tension glaucoma in patients with myopia," Canadian Journal of Ophthalmology, vol. 52, no. 1, pp. 107-113, 2017.

[25] J. Kwon, K. R. Sung, and J. M. Park, "Myopic glaucomatous eyes with or without optic disc shape alteration: a longitudinal study," British Journal of Ophthalmology, vol. 101, no. 12, pp. 1618-1622, 2017.

[26] B. Nuyen, K. Mansouri, and R. N. Weinreb, "Imaging of the lamina cribrosa using swept-source optical coherence tomography," Journal of Current Glaucoma Practice, vol. 6, no. 3, pp. 113-119, 2012.

[27] R. S. Weinreb, J. L. Wheat, and N. V. Rangaswamy, "Age-related losses of retinal ganglion cells and axons," Investigative Opthalmology \& Visual Science, vol. 49, no. 10, pp. 4437-4443, 2008.

[28] L. Wang, G. A. Cioffi, G. Cull et al., "Immunohistologic evidence for retinal glial cell changes in human glaucoma," Investigative Ophthalmology \& Visual Science, vol. 43, no. 4, pp. 1088-1094, 2002.

[29] M. W. Lee, J. M. Kim, Y. I. Shin et al., "Longitudinal changes in peripapillary retinal nerve fiber layer thickness in high myopia: a prospective, observational study," Ophthalmology, vol. 126, no. 4, pp. 522-528, 2018.

[30] R. J. Casson, A. Gupta, H. S. Newland et al., "Risk factors for primary open-angle glaucoma in a Burmese population: the Meiktila Eye Study," Clinical \& Experimental Ophthalmology, vol. 35, no. 8, pp. 739-744, 2007.

[31] J. B. Jonas, P. Martus, and W. M. Budde, "Anisometropia and degree of optic nerve damage in chronic open-angle glaucoma," American Journal of Ophthalmology, vol. 134, no. 4, pp. 547-551, 2002.

[32] J. B. Jonas, K. Ohno-Matsui, and S. Panda-Jonas, "Optic nerve head histopathology in high axial myopia," Journal of Glaucoma, vol. 26, no. 2, pp. 187-193, 2017.

[33] Y. Kimura, M. Hangai, S. Morooka et al., "Retinal nerve fiber layer defects in highly myopic eyes with early glaucoma," Investigative Opthalmology \& Visual Science, vol. 53, no. 10, pp. 6472-6428, 2012.

[34] S. L. Ling, A. J. Chen, U. Rajan, and W. M Cheah, "Myopia in ten year old children--a case control study," Singapore Medical Journal, vol. 28, no. 28, pp. 288-292, 1987.

[35] H. Hashemi, A. Fotouhi, A. Yekta, R. Pakzad, H. Ostadimoghaddam, and M. Khabazkhoob, "Global and regional estimates of prevalence of refractive errors: systematic review and meta-analysis," Journal of Current Ophthalmology, vol. 30, no. 1, pp. 3-22, 2018. 


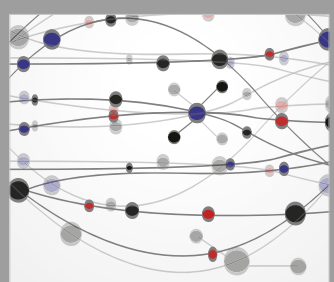

The Scientific World Journal
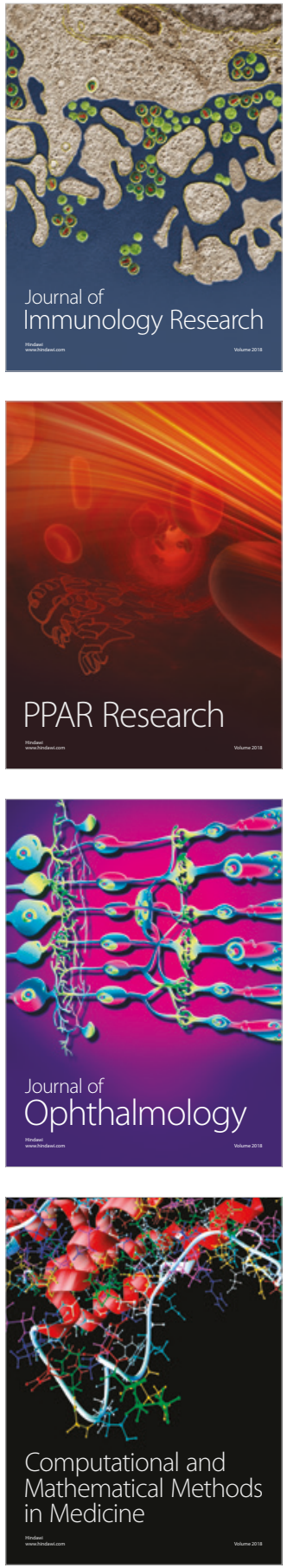

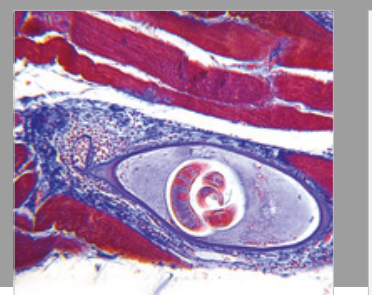

Gastroenterology Research and Practice

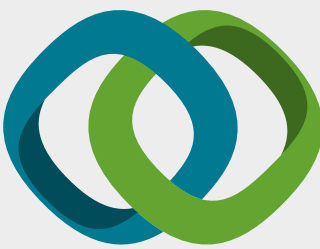

\section{Hindawi}

Submit your manuscripts at

www.hindawi.com
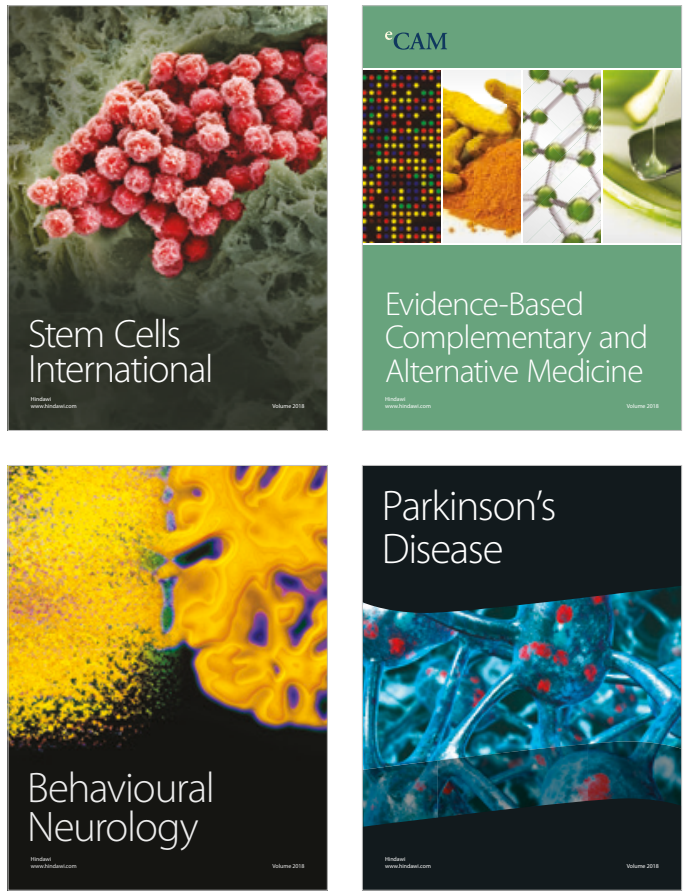

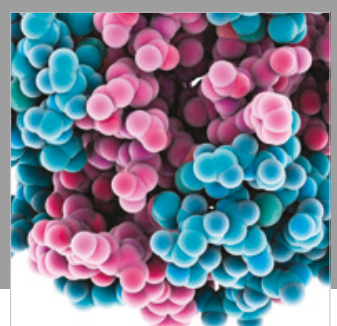

ournal of

Diabetes Research

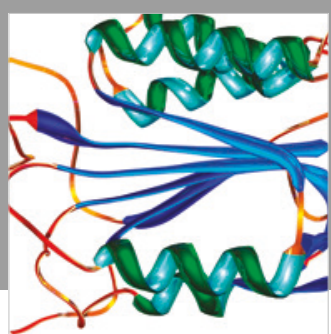

Disease Markers
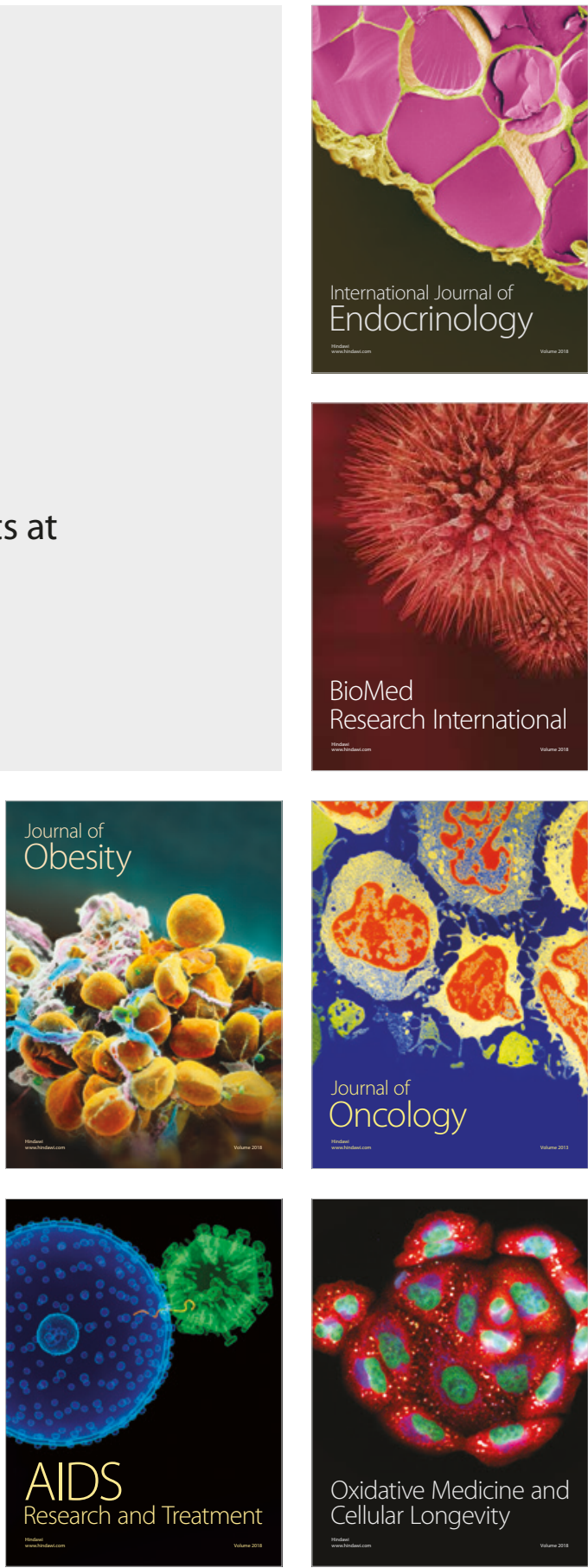